der befallenen Endtriebe von der Krnnenspitze zur Kronenbasis. Pinus strobus wurde bis jetzt nicht befallen, auch dann nicht, wenn sie zwischen Pinus resinosa gepflanzt wurde. Pinus sylvestris, die europäische Wirtspllanze des Wicklers, leidet, obwohl ernste Schädigungen vorkommen hönnen, ebenfalls weniger als Pinus resinosa. Schwerer Befall von Pinus resinosa zeigt sich uagefähr nach 3 Jahren seit der Feststellung der ersten Wickler. Die Larven verursachen Mißbildungen, Zuwachsverlust und Absterben der Terminal- und Seitentriebe. Gemischte Aufforstungen scheinen keinen Schutz zu gewähren. Ebenso gewähren gute Standorte keinerlei Garantie, daß die Pflanzen die Beschädigungen überleben können, solange der Wickler vorhanden ist. Nachdem von den Pinus resinosa-Aufforstungen in Connecticut keine mehr als 33 Jahre als ist. ist man vollkommen im unklaren, ob diese Holzart, nachdem sie eine gewisse Höhe erreicht hat, gegen weitere Beschädigungen gefeit ist. Parasiten, welche von $R$. buoliana im Jahre 1932 gezogen wurden, enthielten 6 Arten, welche als Larvenparasiten von $R$. frustrana Comst. bekannt sind, einen Eiparasiten (Trichogramma sp.) und einen bisher nicht determinierten Larvenparasiten. Alle gezogenen Parasiten sind einheimische Insekten, der Wirt dagegen ein Fxote. Der Parasitismus der Larven betrug im Mai $50 \%$.

In der folgenden Diskussion teilt der Seniorautor mit, daß eine 2 prozent. Lösung von commercial summer oil mit Bleiarsenat (3 lbs. Bleiarsenat auf 100 U. S. gals.) ein befriedigendes Bekämpfungsmittel darstellt. Das Öl tötet die Eier und das Bleiarsenat verhindert die Larven, sich in die Zweige einzufressen. Studien über die Fraßgewohnheiten ergaben, daß 5 Larven 11 Finbohröffnungen an einem Zweig verfortigten. Bezüglich des Transportes von Zierpflanzen wurde festgestellt, daß insbesondere mugho pine ( $P$. mughus) eine Quelle erhöhter Gefahr darstellt. Der Befall an solchen Kiefern kann lange Zeit unentdeckt bleiben.

Dr. Felt teilt mit, daß er durch Spritzen mit Petroloum (light oil), Nikotin und Bleiarsenaten zur Zeit des beginnenden Mottenfluges und ungefähr 2 Wochen später in Aufforstungen von bedeutender Größe und unter natürlichen Bedingungen eine zufriedenstellende Abtötung erreicht habe.

Schedl.

\section{$*$}

Fey, Horst, Wörterbuch der Ungeziefer-, Schädlings-und Pflanzenkrankheitsbekämpfung. Eberswalde, Veriagsgesellschaft R. Müller m. b. H. $212 \mathrm{~s}$.
Der Gedanke, ein Wörterbuch zur Schädlingsbekämpfung zu schaffen, ist neuartig und originell und man kann dem Verfasser auch das Lob spenden, daß er mit viel Fleiß diese Aufgabe angefaßt hat. Das Buch gibt dem Wiederverkäufer von Schädlingsbekämpfungsmitteln die Möglichkeit, sich bei Anfragen seiner Kundschaft in dem Hefte zu unterrichten, wenn freilich auch hier eine etwas konzentriertere Darstellung der Brauchbarkeit des Heftes nicht geschadet hätte. Für den Siedler, dem es der Verfasser ebenfalls in seinem Vorworte empfiehlt, scheint es mir aber ungeeignet zu sein, da es viel zu viel entbält, was den Siedler nicht berührt und ihn deshalb nur verwirrt. Die Stärke dieses Heftes liegt in seiner Zusammenstellung, wenn auch diese vielleicht hätte etwas kritischer geschehen können. (Ob dem Verfasser eigene Erfahrungen auf dem Gebiete der Schädlingsbekämpfung zur Verfügung stehen, die ihn zu dieser Kritik befähigt hätten, entzieht sich meiner Kenntnis.) Für den Siedler hätte der Text mehr beratend gestaltet werden müssen. Auch sind die Angaben über die Bekämpfung mancher Schädlinge - bei den Kellerasseln fiel mir das z. B. auf - ebenso wie bei den Bettwanzen (hier ist die Rolle des Äthylenoxyds z. B. sehr zu kurz gekommen) - nicht immer auf den neuesten Stand unserer Kenntnisse gebracht. Manche für den Siedler gewiß nicht unwichtigen Schädlinge, so z. B. die Maulwurfsgrille, fehlt ganz. Wenn das Thema mit mehr Kritik und wohl auch einiger praktischer Erfahrung behandelt worden wäre, wäre der Text sicherlich von den mancherlei Schlacken bewahrt geblieben. Dr. H. W. lrickhinger-Planegg, Obb.

*

\section{Gartenhaarmüeken als Gerstenschäling.}

Ende April dieses Jahres traten nach R. Schwind (,Kranke Pflanze“ 1935, Nr. 5) auf einem Gerstenfeld (Vorfrucht Kartoffel) bei Gau Algesheim in Rheinhessen die Larven der Gartenhaarmücken stark auf. Die Larven hatten die Gerstenkörner ausgefressen und dadurch etwa ein Drittel des Bestandes vernichtet. Zur Bekämpfung der Gartenhaarmücke wird tiefes Umpflügen der befallenen Felder zur Puppenzeit der Tiere (erste Hälfte Mai) mit darauffolgendem starken Walzen nach Neueinsaat empfohlen. Auch vermehrte Anwendung von Mineraldüngern, insbesondere Kali, hat sich ebenfalls als gute Gegenmaforegel erwiesen. Die erhöhte Kaligabe zwingt die Tiere, tief in den Boden zu gehen, eine gleichzeitige Stickstoffzuführung regt die Pflanzen zu schnellem Wachstum an, so daß sie den Tieren aus dem Rachen wachsen.

\title{
Mitteilungen der Deutschen Gesellschaft für angew. Entomologie E. V.
}

\section{Mitgliederversammlung 1935}

Der Vorstand der Gesellschaft hat beschlossen, die in diesem Jahre fällige Mitgliederversammlung auf Frühahr des nächsten Jahre zu verschieben.

Der Schriftführer: Stellwaag.

\section{Vorstandswahl für die Wahlperiode 1936-1937}

Den Mitgliedern wird in Kürze eine Benachrichtigung darüber zugehen.

Der Schriftführer: Stellwaag. 